\title{
Bistable oscillator dynamics driven by nonwhite noise
}

\author{
L. Fronzoni and P. Grigolini \\ Dipartimento di Fisica, Università degli Studi di Pisa, Piazza Torricelli 2, I-56100 Pisa, Italy \\ Peter Hanggi \\ Department of Physics, Polytechnic Institute of New York, 333 Jay Street, Brooklyn, New York 11201 \\ Frank Moss \\ Department of Physics, University of Missouri at St. Louis, St. Louis, Missouri 63121-4499 \\ R. Mannella and P. V. E. McClintock \\ Department of Physics, University of Lancaster, Lancaster LA1 4 YB, United Kingdom
}

(Received 26 December 1985)

\begin{abstract}
We obtain here an approximate, nonlinear Fokker-Planck-type equation which offers an improved model for two-dimensional nonequilibrium, bistable flows driven by exponentially correlated Gaussian noise. The new model accurately predicts the renormalization of the phase-space statistical densities $P(x, v)$ with correlation time. The theory is tested for accuracy by both analog electronic and digital simulations of a damped oscillator with a bistable potential, driven by additive, colored noise. For large noise strengths, the improved theoretical scheme is applicable for small noise correlation times $\tau$, but becomes increasingly better for small noise strengths where its accuracy extends even to large noise correlation times. The crossover to overdamped motion is also discussed.
\end{abstract}

\section{INTRODUCTION}

The method of modeling the statistical behavior of nonlinear processes which are disturbed by random forces has a long history in a variety of fields. ${ }^{1}$ In most cases one relies on an approximate Markovian theory, e.g., a FokkerPlanck representation for the description of the statistical dynamics of a macroscopic flow. Such an approach, however, implicitly supposes that there exists an extreme separation between the time scales of the macroscopic motion and the fluctuations, so that the latter can be realistically modeled by white ( $\delta$-function-correlated) random forces. On the other hand, there must, of course, always exist corrections to these idealized situations which in certain instances can play an important and observable role. A classic situation where the effect of nonwhite (or colored) noise affects the system behavior in a crucial way is the phenomenon of motional narrowing in magnetic resonance, ${ }^{2}$ where a significant finite correlation time of a fluctuating magnetic field can affect the motion of the corresponding spins significantly.

In recent years, important effects of correlated random forces have been recognized in such fields as activation rates in equilibrium systems $\mathrm{s}^{3-6}$ and driven, stationary nonequilibrium systems, ${ }^{7}$ such as are found, for example, in nonlinear optical systems. ${ }^{8-10}$

The formal theory for dealing with colored noise has been developed to a great extent in recent years. ${ }^{11}$ Approximate schemes, valid for small noise correlation times and small noise intensity, have been well known for some time $e^{12,13}$ and have recently been rediscovered by several groups; $;^{10,14-18}$ and also particularly in the context of the derivation of corrections to the Smoluchowski equation. ${ }^{19}$
Recently, however, several aspects of this "conventional" approximation scheme, which essentially relies on a Taylor-series expansion around the Markovian limit, has come under scrutiny. ${ }^{20,21}$

In the present work, we examine in greater detail the problem of approximation schemes for nonlinear flows driven by time-correlated noise taking as an example the two-dimensional, nonlinear, damped oscillator dynamics.

\section{BISTABLE OSCILLATOR DYNAMICS: THE CONVENTIONAL CORRELATED NOISE APPROXIMATION}

As a typical multidimensional application, let us consider the Brownian motion of a particle of unit mass in a one-dimensional bistable potential field $U(x)$,

$$
U(x)=-\frac{\alpha}{2} x^{2}+\frac{\beta}{4} x^{4}, \alpha>0, \beta>0
$$

and a frictional force $-\gamma \dot{x}$ which is subject to Gaussian, exponentially correlated noise $\xi(t)$, i.e.,

$$
\begin{aligned}
& \dot{x}=v, \\
& \dot{v}=-\frac{\partial U}{\partial x}-\gamma v+\xi(t),
\end{aligned}
$$

where

$$
\langle\xi(t) \xi(s)\rangle=\left(D^{\prime} / \tau\right) \exp (-|t-s| / \tau) .
$$

Using an enlargement of the phase space, this dynamics can be embedded into a three-variable, nonlinear FokkerPlanck dynamics 


$$
\begin{aligned}
& \dot{x}=v, \\
& \dot{v}=-\partial U / \partial x-\gamma v+y, \\
& \dot{y}=-(1 / \tau) y+f_{y}(t)
\end{aligned}
$$

with the white, Gaussian random force

$$
\left\langle f_{y}(t) f_{y}(s)\right\rangle=\left(2 D^{\prime} / \tau^{2}\right) \delta(t-s) .
$$

In order to understand the equivalence of (2.5) with (2.3) we use an integration of (2.4b) with $y(t=0)$ being prepared with the Gaussian stationary probability

$$
\rho_{0}(y)=\left(\tau / 2 \pi D^{\prime}\right)^{1 / 2} \exp \left[-\left(y^{2} \tau / 2 D^{\prime}\right)\right] .
$$

Moreover, the reduced dynamics in (2.2) and (2.3) also depends on the initial preparation scheme between the macrovariables $x(t), v(t)$ and the auxillary variable $y(t) .^{22}$ For relations such as (2.3) to hold true, we must use a "correlation-free" initial preparation; i.e., the initial probability $P_{0}(x, v, y)$ factors as

$$
P_{0}(x, v, y)=P_{0}(x, v) \rho_{0}(y) \text {. }
$$

This then establishes the equivalence between the nonMarkovian dynamics in (2.2) with the enlarged Markovian dynamics in (2.4). ${ }^{20}$

Before we proceed further, we note that the dynamics in (2.2) or (2.4) does not model an equilibrium dynamics obeying detailed balance. This is manifest in $(2.2)$ by the lack of presence of a fluctuation-dissipation relation which would imply a memory damping; i.e., $\gamma \dot{x} \rightarrow \int \gamma(t-s) \dot{x}(s) d s$. For small correlation time $\tau$, the conventional approximation yields an approximate Fokker-Planck equation, which for the system in (2.2) has already been evaluated in the literature. ${ }^{23}$ This reduced, approximate Fokker-Planck equation explicitly reads

$$
\begin{aligned}
\dot{P}_{t}(x, v)= & -v \frac{\partial}{\partial x} P_{t}+\left(\beta x^{3}-\alpha x\right) \frac{\partial}{\partial v} P_{t}+\gamma \frac{\partial}{\partial v}\left(v P_{t}\right) \\
& +D^{\prime} \tau \frac{\partial^{2}}{\partial x \partial v} P_{t}+D^{\prime}(1-\tau \gamma) \frac{\partial^{2}}{\partial v^{2}} P_{t}
\end{aligned}
$$

with the stationary probability $\bar{P}(x, v)$ given by

$$
\bar{P}(x, v)=Z^{-1} \exp \left\{-\gamma\left[U(x)+(1-\tau \gamma)\left(v^{2} / 2\right)\right] / D^{\prime}\right\} .
$$

Thus, the velocity distribution is expected to become more narrow with increasing correlation time $\tau$. More surprising, however, is the prediction that the $x$ distribution, according to the commonly used approximation scheme, would remain unaffected by the finite noise correlation time. In addition, (2.9) exhibits the usual shortcoming that for sufficiently large $\gamma$ the velocity diffusion coefficient takes on negative values.

\section{THE DECOUPLING THEORY}

In an attempt to improve the conventional approximation scheme, let us start from the formally exact equation of motion for the reduced probability $\sigma_{t}(x, v)$. Due to the Gaussian statistics of the correlated noise $\xi(t)$ in (2.2), one can obtain by use of the technique of functional derivatives the exact master equation ${ }^{11}$

$$
\begin{aligned}
\dot{\sigma}_{t}(x, v)= & \frac{\partial}{\partial t}\langle\delta(x(t)-x) \delta(v(t)-v)\rangle \\
= & -v \frac{\partial}{\partial x} \sigma_{t}(x, v)+\left(\beta x^{3}-\alpha x\right) \frac{\partial}{\partial v} \sigma_{t}(x, v)+\gamma \frac{\partial}{\partial v}\left[v \sigma_{t}(x, v)\right] \\
& +\frac{\partial^{2}}{\partial v \partial x}\left[\frac{D^{\prime}}{\tau} \int_{0}^{t} d s \exp \left[-\frac{(t-s)}{\tau}\right]\left\langle\delta(x(t)-x) \delta(v(t)-v) \frac{\delta x(t)}{\delta \xi(s)}\right\rangle\right] \\
& +\frac{\partial^{2}}{\partial v^{2}}\left[\frac{D^{\prime}}{\tau} \int_{0}^{t} d s \exp \left[-\frac{(t-s)}{\tau}\right)\left\langle\delta(x(t)-x) \delta(v(t)-v) \frac{\delta v(t)}{\delta \xi(s)}\right\rangle\right],
\end{aligned}
$$

where the two functional derivatives, $\delta x(t) / \delta \xi(s)$ and $\delta v(t) / \delta \xi(s)$, obey the coupled integral equation

$$
\begin{aligned}
\frac{\delta x(t)}{\delta \xi(s)}=\Theta(t-s) & \left(\int_{s}^{t} \frac{\delta v(r)}{\delta \xi(s)} d r\right) \\
\frac{\delta v(t)}{\delta \xi(s)}=\Theta(t-s) & \left(1+\int_{s}^{t}\left[-\alpha+3 \beta x^{2}(r)\right] \frac{\delta x(r)}{\delta \xi(s)} d r\right. \\
& \left.-\gamma \int_{s}^{t} \frac{\delta v(r)}{\delta \xi(s)} d r\right)
\end{aligned}
$$

Clearly, the master equation in (3.1) is not closed, but in- volves the evaluation of the rather complicated averages inherent in the last two terms. According to the reasoning put forward in a recent paper, ${ }^{21}$ the equation in (3.1) can be closed on a Fokker-Planck level by incorporating a decoupling ansatz between the functional derivatives and the probability

$$
\left.\sigma_{t}(x, v)=\langle\delta(x(t)-x) \delta(v(t)-v))\right\rangle
$$

itself. It should be stressed in this context that by assuming this decoupling approximation, valid for not too large correlation time $\tau$ and small noise strength $D^{\prime}$, one obtains a self-consistent approximation scheme free of addi- 
tional problems. In particular, no non-Fokker-Planck terms (i.e., no differential operators of order $n>2$ ) occur, whose influence on an effective, truncated approximative scheme in full phase space of the type in (2.8) enters in a noncontrollable way. Usually, those infinitely many terms which spoil the Fokker-Planck structure are neglected by relying on nonrigorous, vague arguments only.

In order to shed light on this novel approximation from a different angle and gain additional insight, we will follow here an alternative road based on projector-operator techniques. ${ }^{24}$ The Fokker-Planck operator, which corresponds to the set of stochastic differential equations in (2.4) is divided up into three parts

$$
\mathscr{L}=\mathscr{L}_{a}+\mathscr{L}_{b}+\mathscr{L}_{1} ;
$$

the operator describing the drift,

$$
\mathscr{L}_{a} \equiv-v \frac{\partial}{\partial x}+\left(\beta x^{3}-\alpha x\right) \frac{\partial}{\partial x}+\gamma \frac{\partial}{\partial v} v,
$$

the bath part,

$$
\mathscr{L}_{b}=\frac{1}{\tau} \frac{\partial}{\partial y} y+\frac{D^{\prime}}{\tau^{2}} \frac{\partial^{2}}{\partial y^{2}},
$$

and the interaction part

$$
\mathscr{L}_{1}=-y \frac{\partial}{\partial v} \text {. }
$$

Working within the interaction picture, i.e.,

$$
\widetilde{p}_{t}=\exp \left(-\mathscr{L}_{0} t\right) p_{0}, \quad \mathscr{L}_{0}=\mathscr{L}_{a}+\mathscr{L}_{b}
$$

one obtains for the projected part

$$
\begin{aligned}
& \widetilde{\sigma}_{t}(x, v)=\mathscr{P} \widetilde{p}_{t}(x, v, y), \\
& \mathscr{P} p_{t}(x, v, y)=\rho_{0}(y) \int d y p_{t}(x, v, y)
\end{aligned}
$$

for (3.1) the alternative, exact, formal result $\left[\mathscr{L}_{1}(t)\right.$ $\left.\equiv \exp \left(-\mathscr{L}_{0} t\right) \mathscr{L}_{1} \exp \left(\mathscr{L}_{0} t\right)\right]$

$$
\begin{aligned}
\dot{\sigma}_{t}(x, v)= & \mathscr{P} \mathscr{L}_{1}(t) \widetilde{\sigma}_{t}(x, v) \\
+ & \int_{0}^{t} d s \mathscr{P} \mathscr{L}_{1}(t)\left[\mathscr{T} \exp \left(\int_{s}^{t} d \tau(1-\mathscr{P}) \mathscr{L}_{1}(\tau)\right]\right] \\
& \quad \times(1-\mathscr{P}) \mathscr{L}_{1}(s) \widetilde{\sigma}_{s}(x, v)
\end{aligned}
$$

with $\mathscr{T}$ denoting time ordering. A corresponding inhomogeneous term vanishes identically due to the correlation-free initial preparation (2.7).

In order to proceed, we now introduce three approximations. First, we approximate the time-ordered exponential in (3.7) by the unit operator. Transforming back from the interaction picture we then obtain

$$
\begin{aligned}
\dot{\sigma}_{t}(x, v)= & -v \frac{\partial}{\partial x} \sigma_{t}(x, v)+\left(\beta x^{3}-\alpha x\right) \frac{\partial}{\partial v} \sigma_{t}(x, v) \\
& +\gamma \frac{\partial}{\partial v}\left[v \sigma_{t}(x, v)\right] \\
& +\frac{D^{\prime}}{\tau} \int_{0}^{t} d s \frac{\partial}{\partial v}\left[\exp \left[\mathscr{L}_{a}-\frac{1}{\tau}\right)(t-s)\right] \\
& \times \frac{\partial}{\partial v} \sigma_{t}(x, v) .
\end{aligned}
$$

Next we use, inside the last term in (3.8), the identity ${ }^{25,26}$

$$
\exp \left(\mathscr{L}_{a} t\right) \frac{\partial}{\partial v}=\exp \left(\mathscr{L}_{a}^{x} t\right) \frac{\partial}{\partial v} \exp \left(\mathscr{L}_{a} t\right),
$$

where $\mathscr{L}_{a}^{x} A \equiv \mathscr{L}_{a} A-A \mathscr{L}_{a}$. Further we employ the linear-response approximation, that is, we shall use within this last term, the approximation

$$
\exp \left(\mathscr{L}_{a}^{t}\right) \sigma_{t}(x, v)=\sigma_{t}(x, v)
$$

By use of the two relations

$$
\mathscr{L}_{a}^{x} \frac{\partial}{\partial v} \equiv \frac{\partial}{\partial x}-\gamma \frac{\partial}{\partial v}
$$

and

$$
\mathscr{L}_{a}^{x} \frac{\partial}{\partial x}=\left(\alpha-3 \beta x^{2}\right) \frac{\partial}{\partial v},
$$

our third approximation consists in the replacement of (3.11b) by the effective operator approximation

$$
\mathscr{L}_{a}^{x} \frac{\partial}{\partial x} \simeq\left(\alpha-3 \beta\left\langle x^{2}\right\rangle\right) \frac{\partial}{\partial v} .
$$

This latter approximation precisely mirrors the decoupling ansatz put forth in Ref. 21. Thus we end up with a closed, approximate, effective Fokker-Planck equation

$$
\begin{aligned}
\dot{\sigma}_{t}(x, v)= & -v \frac{\partial}{\partial x} \sigma_{t}(x, v)+\left(\beta x^{3}-\alpha x\right) \frac{\partial}{\partial v} \sigma_{t}(x, v)+\gamma \frac{\partial}{\partial v}\left[v \sigma_{t}(x, v)\right] \\
& +\frac{D^{\prime}}{1+\gamma \tau+\left(3 \beta\left\langle x^{2}\right\rangle-\alpha\right) \tau^{2}} \frac{\partial^{2}}{\partial v^{2}} \sigma_{t}(x, v)+\frac{D^{\prime} \tau}{1+\gamma \tau+\left(3 \beta\left\langle x^{2}\right\rangle-\alpha\right) \tau^{2}} \frac{\partial^{2}}{\partial x \partial v} \sigma_{t}(x, v) .
\end{aligned}
$$

Its stationary probability $\bar{\sigma}(x, v)$ is found to be

$$
\begin{aligned}
\bar{\sigma}(x, v)= & Z^{-1}\left[\exp \left(-\frac{1}{2} v^{2} / \sigma_{v}\right)\right] \\
& \times\left\{\exp \left[-\left(-\alpha x^{2} / 2+\beta x^{4} / 4\right) / \sigma_{x}\right]\right\}
\end{aligned}
$$

with

$\sigma_{x}=\left(D^{\prime} / \gamma\right)\left[1+\tau\left(3 \beta\left\langle x^{2}\right\rangle-\alpha\right) /(\gamma+1 / \tau)\right]^{-1}$,

$\sigma_{v}=\left(D^{\prime} / \gamma\right)\left[1+\tau \gamma+\tau^{2}\left(3 \beta\left\langle x^{2}\right\rangle-\alpha\right)\right]^{-1}$.
The following features of this decouplingapproximation scheme are evident. Because $\left\langle x^{2}\right\rangle \simeq \alpha / \beta$, the effective diffusion coefficients occurring in (3.13) are non-negative, i.e., no artificial boundaries are exhibited. Moreover, the stationary probability $\bar{\sigma}(x, v)$, becomes more narrow with increasing noise correlation time not only in the velocity coordinate $v$, but also in the configuration coordinate $x$. That $\bar{\sigma}(x)$ is narrowed with increasing $\tau$ has already been predicted and observed for a two- 
dimensional system represented by the overdamped limit of Eq. (2.2) with (2.1) by the results of Hanggi et al., ${ }^{21}$ Jung and Risken, ${ }^{27}$ and experimentally by Moss and McClintock. ${ }^{28}$ The results of the conventional perturbative scheme in (2.8) and (2.9) are recovered from (3.13) and (3.14) if we set equal to zero the effective nonlinearity, $3 \beta\left\langle x^{2}\right\rangle-\alpha=0$, and expand in first order of the noise correlation time $\tau$.

\section{DIFFUSION EQUATION \\ FOR THE CONFIGURATION COORDINATE}

Finally, we discuss here the implications of our novel approximation for the overdamped case, where the configuration coordinate remains the only slowly varying observable. The standard approximation schemes $^{1(\mathrm{a}), 10,12-18,29}$ are recovered if we were to expand the exponent in (3.8) in a power series. In contrast, the important property of the approximation in (3.12), yielding the potential renormalization in (3.14), as a function of the correlation time $\tau$, essentially corresponds to a resummation of infinitely many terms $\sim \tau^{n}$. This very feature is evident if we contract the dynamics onto the $x$ variable alone.

For example, let us integrate (2.9) over the velocity variable. This leaves one with a stationary probability for $x$, which is not dependent on $\tau$. This is in clear contrast with the result in Ref. 21 . This readily can be understood if we recall that (2.8) is based on a small- $\tau$ expansion $(\tau \rightarrow 0)$, such that $\tau \gamma<<1$. In the overdamped limit $(\gamma \rightarrow \infty),(2.8)$ no longer remains in the range of useful applicability, because $\tau \gamma \gg 1$ for $\tau$ small, but finite [see also the comments made after Eq. (7.8) of Ref. 10(a)].

On the contrary, if we are going to perform a contraction over the velocity $v$ in the improved approximation (3.13) we obtain for the reduced probability $\sigma_{t}(x)$,

$$
\sigma_{t}(x) \equiv \int \sigma_{t}(x, v) d v
$$

an effective Fokker-Planck equation, where with $a=\alpha / \gamma$, $b=\beta / \gamma$, and $D=D^{\prime} / \gamma^{2}$,

$$
\begin{aligned}
\dot{\sigma}_{t}(x)= & {\left[-\frac{\partial}{\partial x}\left(a x-b x^{3}\right) \sigma_{t}(x)\right] } \\
& +\frac{D[1+1 /(\gamma \tau)]}{1+1 / \gamma \tau+\tau\left(3 b\left\langle x^{2}\right\rangle-a\right)} \frac{\partial^{2}}{\partial x^{2}} \sigma_{t}(x) .
\end{aligned}
$$

In the overdamped limit, i.e., $\gamma \tau \gg 1$, we recover from (4.2) precisely the limiting result obtained by Hanggi et al., 21

$$
\begin{aligned}
\dot{\sigma}_{t}^{\text {over }}(x)= & {\left[-\frac{\partial}{\partial x}\left(a x-b x^{3}\right) \sigma_{t}(x)\right] } \\
& +\frac{D}{1+\tau\left(3 b\left\langle x^{2}\right\rangle-a\right)} \frac{\partial^{2}}{\partial x^{2}} \sigma_{t}(x)
\end{aligned}
$$

This is clear evidence that the novel theory, based on (3.10) and (3.12), has advantageous features; it is quite different from an expansion in small $\tau$ of $(2.8)$, which is not compatible with $\gamma \tau \gg 1$.

\section{EXPERIMENTAL TESTS}

The main result of this new approximate approach is the prediction, given by Eqs. (3.13) and (3.14), that the noise correlation time not only reshapes the density of the velocity coordinate, but also that of the displacement. Such effects ought to be easily observable. In addition, such an experiment also would critically check the limits of applicability of our novel, but still approximative, theory.

In this section we report the results of separate experiments, carried out in Pisa, Lancaster, and St. Louis, on quite different electronic circuits which, however, effectively mimic the three-dimensional dynamics defined by Eqs. (2.2) and (2.4). Our conclusions are reinforced by a digital simulation which is discussed below. All these results confirm the predictions of Eqs. (3.14). It is also important to note that similar such effects have recently been predicted by matrix continued-fraction solutions of a two-dimensional Fokker-Planck equation ${ }^{27}$ and observed ${ }^{28}$ in measurements of $P(x, y)$ for an overdamped system.

The experimental approach is similar to that used in previous work..$^{30,31}$ Electronic analogs of Eq. (2.1) and (2.2) were constructed, driven by exponentially correlated Gaussian noise, and the fluctuating output voltages were digitized and analyzed by computer. The circuits are shown in Fig. 1. The construction and functioning of the

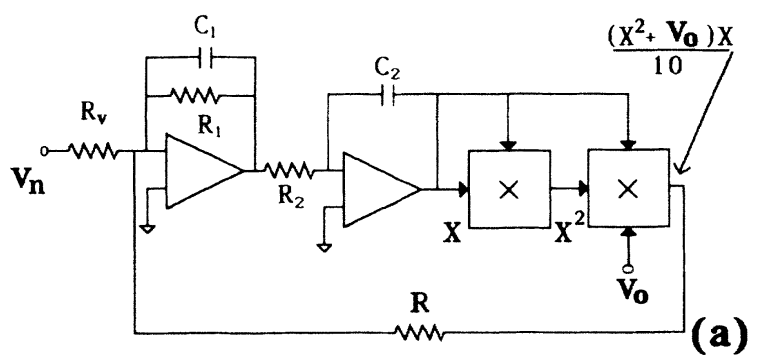

$$
\ddot{X}=-\frac{\dot{X}}{R_{1} C_{1}}+\frac{V_{\mathbf{O}} X}{10 R C_{1} R_{2} C_{2}}-\frac{X^{3}}{10 R C_{1} R_{2} C_{2}}+\frac{V_{n}}{R_{\mathbf{v}} C_{1} R_{2} C_{2}}
$$

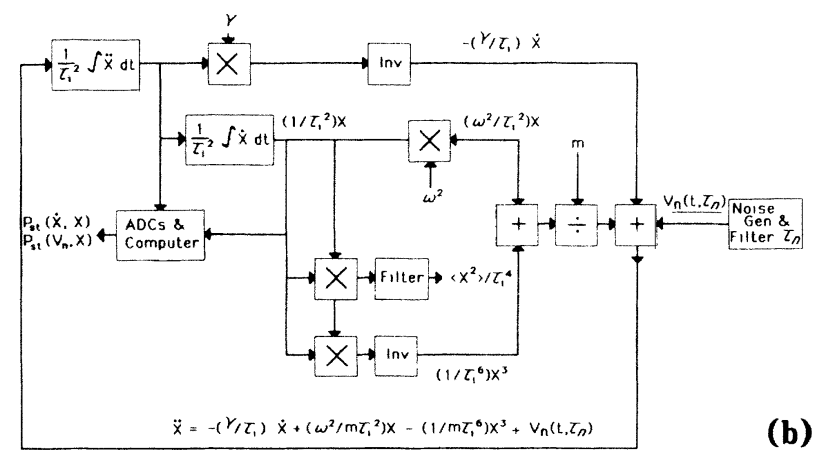

FIG. 1. The analog simulators. (a) The "minimum component" system used in Pisa, (b) the system used in Lancaster and St. Louis. Both systems are constructed from standard ana$\log$ electronic components. Multipliers, dividers, and summers are indicated by the appropriate arithmetic symbol. 
circuit used in Pisa has been discussed elsewhere ${ }^{31}$ and so will not be detailed here. The two simulators are quite different from the points of view of design (the circuit used in Pisa is a "minimum component" design) and internal time scaling. Actual measurements of the rms noise voltage and correlation time were scaled to dimensionless values in accord with Eq. (2.3), which for $t \rightarrow s$ results in $\left\langle\xi^{2}\right\rangle=D^{\prime} / \tau$. In this relation, $\tau$ is the dimensionless noise correlation time defined by $\tau=\tau_{n} / \tau_{i}$, where $\tau_{n}$ is the actual correlation time of the noise voltage applied to the simulator and $\tau_{i}$ is the internal time scale of the simulator defined by the integrator time constant. The colored-noise voltages were prepared in the usual way by passing wide-band (approximately white) noise through a low-pass, single-pole filter, whose cutoff frequency determined $\tau_{n}$. In the circuit used in St. Louis and Lancaster $\tau_{i}=100 \mu \mathrm{s}$ and $\tau_{n}$ varied from $50 \mu \mathrm{s}(\tau=0.5)$ to $200 \mu \mathrm{s}$ $(\tau=2)$. The circuit used in Pisa, by contrast, functioned with an internal time scale approximately 1 order of magnitude larger, but was operated over the same range of dimensionless parameters.

We display first the data from Pisa, which were obtained for small noise intensity $D^{\prime}<0.5$. In Fig. 2 are shown the measured values of $P(x)$ for a range of values of $\tau$. It is unmistakable that the shape of $P(x)$ is significantly altered by $\tau$, the ratio $R$ of the maxima to the minimum varying from $R \simeq 3.2$ for the smallest $\tau$ to $R \simeq 19$ for the largest. The solid curves in Fig. 2 were obtained from Eqs. (3.14) after integrating over all velocities. The only adjustable parameter used to fit the data was the normalization constant of the density. This constant was obtained simply by requiring that the calculated and measured maxima be approximately equal. Under this condition, a rather strong test of the theory is obtained by observing how well the minima in $P(x)$ are then predicted. Figure 2 shows that the new approximate theory is surprisingly accurate for small $D^{\prime}$, even for rather large $\tau$.

Figure 3 shows the measured Pisa velocity distributions compared to Eqs. (3.14), integrated over all displacements. As before, the normalization constant was obtained by matching theory to measurement at the maxima, so that the predicted widths serve as tests of the accuracy.

We have also made a digital simulation of Eq. (2.2) with (2.1) and (2.3) using standard techniques. Equation (2.2) was integrated using the predictor method, ${ }^{32}$ with a time step of $\Delta t=10^{-2}$ for $4 \times 10^{6}$ points chosen at random from a table of Gaussian numbers of the desired standard deviation. The finite correlation time was built in by allowing each chosen noise point to decay (for a number of iterations) at a rate chosen from an exponential distribution. Both the Gaussian nature and the exponential dependence of the correlations have been tested, the former by comparing simulated and calculated moments up to the eighth order, and the latter by insisting that the fits to an exponential function be accurate to a few percent.

The digital simulations were all carried out for $D^{\prime}=1$ and $\gamma=1$ for $\tau$ varying from 0.1 to 1.0. The results are shown in Fig. 4 by the vertical bars compared to the theory shown by the curves. Two remarks are obvious: first, that the agreement between theory and simulation is
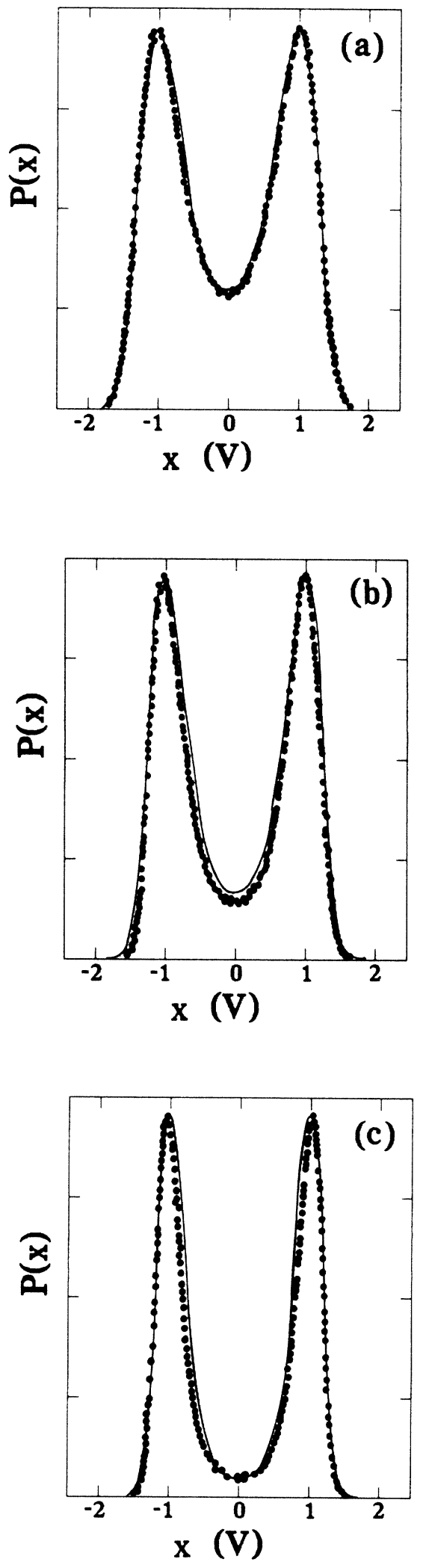

FIG. 2. The analog measurements taken in Pisa (solid circles) of $P(x)$ compared to the predictions of Eqs. (3.14) (solid curve) for $D=0.32$ and $\gamma=1.0$; (a) $\tau=0.63$, (b) $\tau=1.17$, and (c) $\tau=2$.14. Statistical measurement errors are indicated by the size of the circles. 

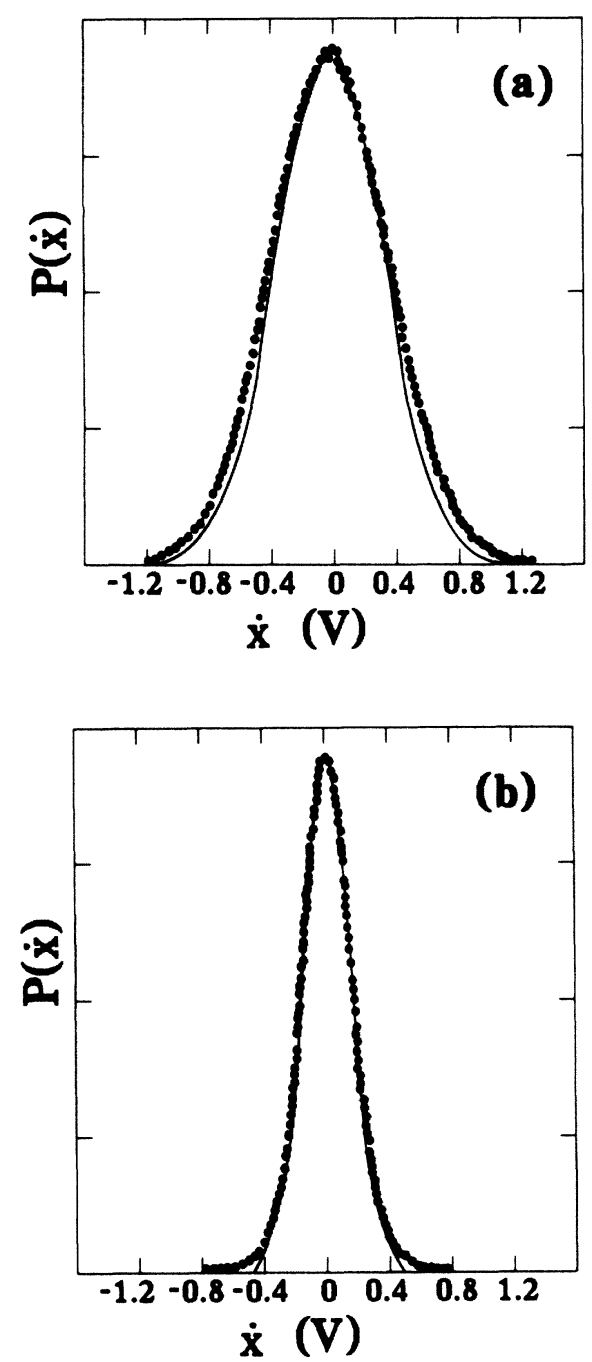

FIG. 3. The analog measurements taken in Pisa (solid circles) of $P(\dot{x})$ compared to the predictions of Eqs. (3.14) (solid curve) for $D=0.32$ and $\gamma=1.0$; (a) $\tau=0.63$ and (b) $\tau=2.14$. The values of $\dot{x}$ were measured in volts as shown, but are numerically equal to the velocities given by Eqs. (3.14).

satisfactory only for the smallest $\tau(\tau=0.1)$; and second, despite the poor agreement, it is nevertheless quite clear that the shape of $P(x)$ is changed in at least qualitative agreement with the data obtained at Pisa. The question is whether the theory becomes inaccurate at large $D^{\prime}$ (and large $\tau$ ) or whether the digital simulation is at fault. We show below, with the circuit used in St. Louis and Lancaster, that it is the former.

The results of measurements carried out on this simulator are shown in Fig. 6 for small $D^{\prime}\left(D^{\prime}\right.$ chosen equal to that of the Pisa measurements). The predictions of the theory are shown by the smooth curves. Clearly, satisfactory agreement has been demonstrated among the theory and the two experiments for $D^{\prime}=0.32$. However, for larger $D^{\prime}=1$, (corresponding to the digital simulation) measurements of $P(x)$ made on this simulator, shown in Fig. 5, are in quite good agreement with the digital simu-
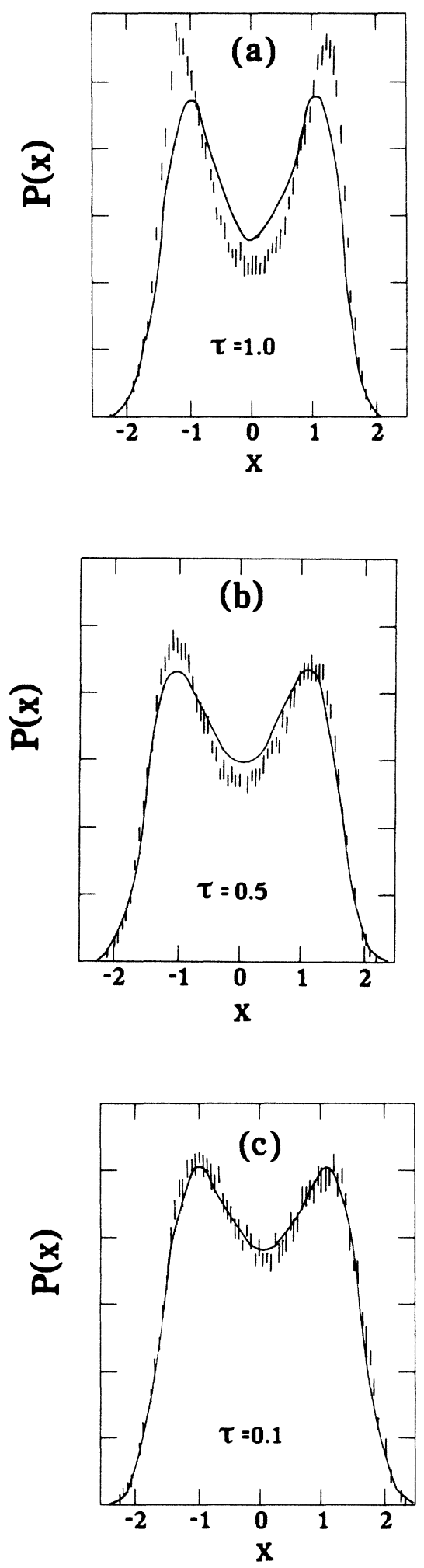

FIG. 4. The digital simulations of $P(x)$ done in Lancaster (vertical bars) compared to Eqs. (3.14) (solid curve) for (a) $\tau=1.0$, (b) $\tau=0.5$, and (c) $\tau=0.1$. All simulations were for $D=1$ and $\gamma=1$. The accuracy of the simulation is indicated by the height of the bars. 


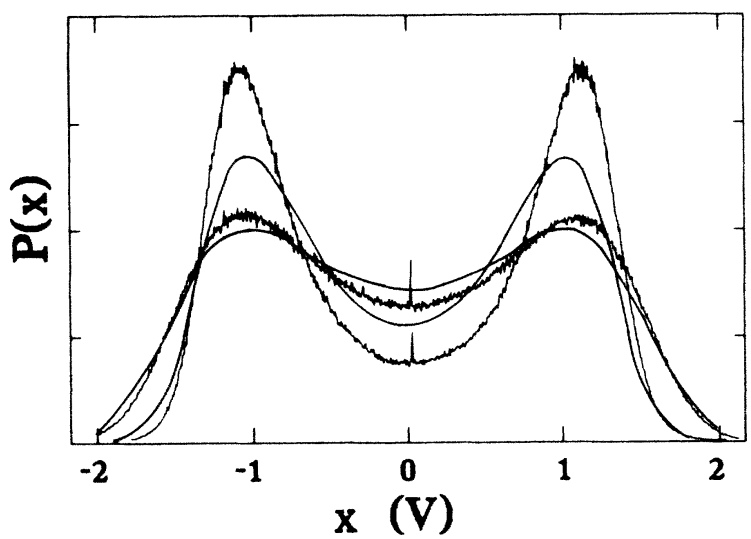

FIG. 5. The analog measurements done at Lancaster and St. Louis (jagged curves) of $P(x)$ compared to Eqs. (3.14) (smooth curves) for large noise intensity: $D=1$ and $\gamma=1$; for $\tau=0.5$ (shallower curves) and $\tau=2.0$ (steeper curves).

lations. We therefore conclude that the theory becomes inaccurate for noise strengths approaching $D^{\prime}=1$.

\section{CONCLUSIONS}

It is not difficult to conclude that the new colored-noise approximation, obtained here by the projector-operator method and previously by use of the functional derivative method, ${ }^{21,9}$ represents a profound improvement over the conventional approximate method. It has been shown by both digital and analog simulation that the new approximation is quite accurate for large noise correlation times, though the upper limit of the accuracy is not known. The approximation is, however, only good near the white-noise limit $(\tau \sim 0.1)$ for large noise intensity $\left(D^{\prime} \sim 1\right)$, but regains its accuracy for large $\tau$ at small noise intensity $\left(D^{\prime}<0.3\right)$.

We might also point out that, while we had previously tested this approximation on a two-dimensional system

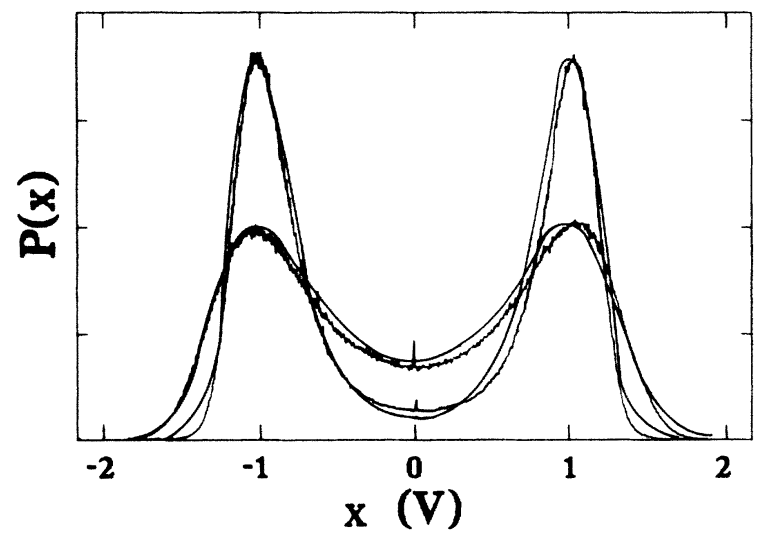

FIG. 6. The analog measurements done in Lancaster and St. Louis (jagged curves) of $P(x)$ compared to Eqs. (3.14) (smooth curves) for small noise intensity: $D=0.32$ and $\gamma=1$; for $\tau=0.5$ (shallow broad curves) and $\tau=2.0$ (steep narrow curves).

[the overdamped limit of Eq. (2.2)] by making sojourn time measurements, ${ }^{21}$ we have here demonstrated its efficacy on a three-dimensional system, see Eqs. (2.4). Moreover, we have here measured the densities which are the more fundamental quantities representing direct, stationary solutions of the Fokker-Planck equation. We hope that our theory proves to be useful in describing multidimensional nonlinear optical systems for which correlated noise necessarily plays an essential role. ${ }^{8,9}$

\section{ACKNOWLEDGMENTS}

We are grateful to Professor Hans Risken for a stimulating discussion on higher-dimensional systems driven by colored noise. This work was supported in part by grants from the U.S. Office of Naval Research (No. ONR N00014-85-K-0372) and the U.K. Science and Enginering Research Council.
${ }^{1}$ For a set of review articles, see R. L. Stratonovich, Topics in the Theory of Random Noise (Gordon and Breach, New York, 1963), Vol. I; Vol. II (1967); H. Haken, Rev. Mod. Phys. 47, 67 (1975); G. Nicolis, Rep. Prog. Phys. 42, 225 (1979); P. Hanggi and H. Thomas, Phys. Rep. 88c, 207 (1982); H. Risken, The Fokker-Planck Equation, Vol. 18 of Springer Series in Synergetics (Springer, New York, 1984); and the various contributions in Fluctuations and Sensitivity in Nonequilibrium Systems, Vol. I of Springer Proceedings in Physics, edited by W. Horsthemke and D. K. Kondepudi (Springer, New York, 1984).

${ }^{2}$ R. Kubo, in Fluctuations, Relaxation and Resonance in Magnetic Systems, edited by D. ter Harr (Oliver and Boyd, Edingburgh, 1962), p. $23 \mathrm{ff}$.

${ }^{3}$ R. F. Grote and J. T. Hynes, J. Chem. Phys. 73, 2715 (1980); 77, 3736 (1982).
${ }^{4}$ P. Hanggi and F. Mojtabai, Phys. Rev. A 26, 1168 (1982); J. Stat. Phys. 30, 401 (1983); P. Hanggi and U. Weiss, Phys. Rev. A 29, 2265 (1984).

${ }^{5}$ B. Carmeli and A. Nitzan, Phys. Rev. A 29, 1481 (1984).

6(a) P. Hanggi, J. Stat. Phys. 42, 105 (1986); (b) T. Fonseca, J.A.N.F. Gomes, P. Grigolini, and F. Marchesoni, Adv. Chem. Phys. 62, 389 (1985).

${ }^{7}$ P. Hanggi and P. Riseborough, Phys. Rev. A 27, 3379 (1983); C. Van den Broeck and P. Hanggi, ibid. 30, 2730 (1984).

${ }^{8}$ R. Short, L. Mandel, and R. Roy, Phys. Rev. Lett. 49, 647 (1982); R. F. Fox, G. E. James, and R. Roy, Phys. Rev. A 30, 2482 (1984).

${ }^{9} \mathrm{P}$. Hanggi and F. Moss, in Proceedings of the Conference on Instabilities in Lasers and Nonlinear Optical Systems, edited by R. W. Boyd, M. G. Raymer, and L. M. Narducci (Cambridge University, Cambridge, 1986). 
10(a) A. Schenzle and T. Tel, Phys. Rev. A 32, 596 (1985); (b) A. Schenzle and R. Graham, Phys. Lett. 98A, 319 (1983).

${ }^{11}$ The general theory for Gaussian and non-Gaussian, correlated random forces together with a discussion of exactly solvable cases can be found in the following: (a) P. Hanggi, Z. Phys. B 31, 407 (1978); (b) in Stochastic Processes Applied to Physics, edited by L. Pesquera and M. Rodriguez (World Scientific, Singapore, 1985), pp. 69-95.

${ }^{12}$ R. L. Stratonovitch, Ref. 1. Vol. I.

${ }^{13}$ M. Lax, Rev. Mod. Phys. 38, 541 (1966).

${ }^{14}$ N. G. Van Kampen, Phys. Rep. 24C, 171 (1976).

15J. M. Sancho, M. San Miguel, S. L. Katz, and J. D. Gunton, Phys. Rev. A 26, 1589 (1982); K. Lindenberg and B. J. West, Physica 119A, 485 (1983); 128A, 25 (1984).

${ }^{16}$ H. Dekker, Phys. Lett. 90A, 26 (1982).

${ }^{17}$ R. F. Fox, Phys. Lett. 94A, 281 (1983); J. Stat. Phys. 16, 259 (1977).

${ }^{18}$ L. A. Lugiato and R. J. Horowicz, J. Opt. Soc. Am. 2, 971 (1985).

${ }^{19}$ (a) G. Wilemski, J. Stat. Phys. 14, 153 (1976); (b) J. L. Skinner and P. G. Wolynes, Physica A96, 561 (1979); (c) S. Chartuverdi and S. Shibata, Z. Phys. B 35, 297 (1979), (d) H. Risken, H. D. Vollmer, and M. Morsch, ibid. 40, 343 (1980); (e) F. Haake, ibid. 50, 71 (1982); (f) F. Marchesoni and P. Grigolini, Physica A121, 169 (1983); for a very recent review on this topic see (g) P. Grigolini and F. Marchesoni, Adv. Chem. Phys. 62, 29 (1985).

${ }^{20}$ P. Hanggi, F. Marchesoni, and P. Grigolini, Z. Phys. B 56, 333 (1984).

${ }^{21}$ P. Hanggi, T. J. Mroczkowski, F. Moss, and P. V. E. McClintock, Phys. Rev. A 32, 695 (1985).

${ }^{22}$ H. Grabert, P. Hanggi, and P. Talkner, J. Stat. Phys. 22, 537 (1980).
${ }^{23}$ See A. Schenzle and T. Tel, Ref. 10(a), Eqs. (7.1)-(7.4) and (3.9); and/or N. G. Van Kampen, Ref. 14, p. 214.

${ }^{24}$ P. Grigolini, Adv. Chem. Phys. 52, 1 (1985).

${ }^{25}$ P. Grigolini and F. Marchesoni, Ref. 19(g), p. 59.

${ }^{26}$ L. T. Muus, in Electron Spin Relaxation in Liquids, edited by P. W. Atkins (Plenum, New York, 1972); p. 4ff.

${ }^{27}$ P. Jung and H. Risken, Z. Phys. B 61, 367 (1985).

${ }^{28}$ F. Moss and P. V. E. McClintock, Z. Phys. B 61, 381 (1985).

${ }^{29}$ P. Grigolini and F. Marchesoni, Adv. Chem. Phys. 62, 29 (1985).

30(a) J. Smythe, F. Moss, and P. V. E. McClintock, Phys. Rev. Lett. 51, 1062 (1983); (b) J. Smythe, F. Moss, and P. V. E. McClintock, Phys. Lett. 97A, 95 (1983); (c) F. Moss, in Proceedings of the Workshop on Chaos in Nonlinear Dynamical Systems, edited by J. Chandra (SIAM, Philadelphia, 1984), Chap. X; (d) J. Robinson, F. Moss, and P. V. E. McClintock, J. Phys. A 18, L89 (1985); (e) P. V. E. McClintock and F. Moss, Phys. Lett. 107A, 367 (1985); (f). J. M. Sancho, R. Mannella, P. V. E. McClintock, and F. Moss, Phys. Rev. A 32, 3639 (1985).

${ }^{31}$ (a) L. Fronzoni, in Advances in Non-Linear Dynamic and Stochastic Processes edited by R. Livi and A. Politi (World Scientific, Singapore, 1985), p. 201; (b) S. Faetti, C. Festa, L. Fronzoni, P. Grigolini, and P. Martano, Phys. Rev. A 30, 3252 (1984); (c) C. Festa, L. Fronzoni, P. Grigolini, and F. Marchesoni, Phys. Lett. 102A, 95 (1984); (d) S. Faetti, C. Festa, L. Fronzoni, and P. Grigolini, in Memory Function Approaches to Stochastic Problems in Condensed Matter, edited by M. W. Evans, P. Grigolini, and G. Pastori Paravicini (Wiley, New York, 1985), Vol. 62, p. 445.

${ }^{32}$ M. Klerer and G. Korn, Digital Computer Users Handbook (McGraw-Hill, New York, 1967), Chap. 2.6. 